Revue française de la traduction

\title{
Intraduisibles et intraduits, des sciences humaines aux textes littéraires
}

\section{Claire Placial}

\section{OpenEdition}

\section{Journals}

Édition électronique

URL : http://journals.openedition.org/traduire/706

DOI : $10.4000 /$ traduire.706

ISSN : 2272-9992

\section{Éditeur}

Société française des traducteurs

\section{Édition imprimée}

Date de publication : 15 juin 2015

Pagination : 115-127

ISSN : 0395-773X

\section{Référence électronique}

Claire Placial, «Intraduisibles et intraduits, des sciences humaines aux textes littéraires », Traduire [En ligne], 232 | 2015, mis en ligne le 15 juin 2017, consulté le 01 mai 2019. URL : http:// journals.openedition.org/traduire/706 ; DOI : 10.4000/traduire.706 


\section{Intraduisibles et intraduits, des sciences humaines aux textes littéraires}

\section{Claire Placial}

La question des intraduisibles est un éternel serpent de mer, sinon de la traduction (puisque tout de même on traduit), du moins de la traductologie. La question que pose le présent article est la suivante : les " intraduits " (j'entends par ce terme un mot ou une expression laissée en langue originale dans la traduction) et les " intraduisibles " sont-ils de même nature dans les textes littéraires et dans les textes de sciences humaines ? S'il est fréquent que les traductions de textes de sciences humaines conservent une partie du lexique de l'œuvre source, on entend souvent que la non-traduction, en traduction littéraire, est un échec du traducteur. Mon propos est de poser quelques limites à cette distinction qui définit le texte, sinon par son entière traductibilité intrinsèque, du moins par l'impératif de tout traduire, à partir d'une étude de cas confrontant des textes traduits en français de l'italien, et faisant une large place aux dialectes, régionalismes ou idiosyncrasies des personnages. Partons d'un exemple quelque peu radical, celui des traductions effectuées par Serge Quadruppani des romans du sicilien Andrea Camilleri. Ainsi ces quelques lignes de dialogue, dans Le tour de la bouée, entre le commissaire Montalbano et le pêcheur Ciccio Albanese, qui évoque un vieux marin, Stefano Lagumina :

- Stefano Lagumina, mais tout le monde l'appelle 'u zu, l'oncle Stefanu, il a 85 ans, avé la tête plus claire que c'est pas possible. (...) Et 'u zu est d'accord avec mia, avec moi.

- Et quelles sont vos conclusions?

- Attendez, vegnu e mi spiegu, je vais m'expliquer. (Camilleri traduit par Quadruppani 2005 : p. 70)

Le moins que l'on puisse dire, c'est que subsistent dans le texte français bon nombre de mots italiens, ou plutôt, siciliens. Le traducteur littéraire n'a pas tout traduit. Certes Quadruppani se fait le chantre d'une traduction littéraire qui casse les codes de la belle langue française, et en cela il s'oppose à la tradition de traduction visant à produire une traduction qui donne l'impression que le texte original ait été directement écrit en français. Pour autant, les traducteurs littéraires n'ont pas attendu Quadruppani pour laisser subsister dans le texte français des traces du texte de départ. Mais avant d'en donner quelques exemples, posons la question 
suivante : si les traductions françaises de textes littéraires italiens du siècle dernier laissent fréquemment subsister des mots ou phrases du texte source, est-ce que pour autant ces " intraduits " sont des intraduisibles ? Qu'est-ce qui, pour le traducteur, mène à ne pas traduire, ou bien encore, comme le fait Quadruppani dans l'extrait que je viens de citer, à reproduire une expression sicilienne puis à la gloser? Mon postulat est que, selon les cas, il ne s'agit pas tant d'un échec de la traduction que d'une affirmation de son rôle d'intermédiaire, révélant la nature fondamentalement étrangère du texte source. Mais c'est singulièrement lorsque le texte source se fait lui-même en partie étranger que la traduction française elle-même laisse des intraduits. Vegnu e mi spiegu, comme dit le personnage de Camilleri, je m'explique : singulièrement, ce n'est pas autour d'une notion particulièrement résistante que le texte ne traduit pas, pas pour une question sémantique donc, mais plutôt lorsque l'ouvrage littéraire se fait polyphonique par l'utilisation de dialectes, de langues étrangères, de citations. L'hétérogénéité linguistique et stylistique du texte source appelle alors une hétérogénéité isomorphe, si l'on peut dire, dans le texte français. C'est ce que cet article examinera, à travers des exemples de traduction de textes littéraires italiens rédigés dans un entrelacs d'italien littéraire, de dialectes, de langues étrangères.

\section{Une réponse littéraire : de la transposition à la recréation}

Considérons d'abord des traductions qui semblent répondre à l'injonction de " tout traduire ", en élaborant des solutions qui permettent de reproduire, avec les ressources de la langue française, l'hétérogénéité linguistique du texte de départ. Trois exemples à ce titre, qui illustrent une évolution des possibilités prêtées par les traducteurs à la langue française.

D'abord le cas Gadda. Les romans de Carlo Emilio Gadda font largement usage des dialectes italiens, qui sont parfois entremêlés : l'intérêt de ce travail dépasse celui d'un ancrage sociologique ou géographique et révèle en réalité des conceptions très particulières du maniement littéraire de la langue. Traduire, dans ces conditions, est un défi de taille. Voici un court extrait, qui donne la parole à l'inspecteur Francesco Ingravallo, l'enquêteur de L'Affreux pastis de la rue des Merles, un roman de 1957, traduit par Louis Bonalumi en 1963 :

Cosi proprio cosi, avveniva dei "suoi " delitti. "Quanno me chiammeno!... Già. Si me chiammeno a me... puo sta ssicure ch'è nu guaio: quacche gliummero... de sberretà.... diceva, contaminando napolitano, molisano, e italiano. (Gadda, 1957 : p. 7)

C'était pareil, tel que, pour " ses " forfaits. "Quand ils me 'eulent. . . 'ouais ", disait-il charabiant napolitain, molisan, italien, "quand ils " "iennent se chercher bibi... tu peux " tre sûr qu'y a du vilain : un pot-aux-roses en perspective... à dépiauter... ". (Gadda traduit par Bonalumi, 1963 : p. 13)

La traduction de Bonalumi traduit littéralement l'incise, ainsi apprenons-nous qu'Ingravallo mélange à son italien des traits molisans et napolitains. Mais là où le lecteur italien reconnaît 
ces dialectes, là où ailleurs dans le roman il entend le dialecte ou plutôt l'accent romain jusque dans le titre du roman, Quer pasticciaccio brutto et non Quel - le lecteur français en revanche ne peut les reconnaître. Le traducteur substitue en effet assez systématiquement aux dialectes une langue française populaire, orale, qui penche plutôt du côté des inflexions d'un marlou parisien que de celles d'un commissaire molisan. Pour autant Bonalumi cherche, dans une certaine mesure, à utiliser des ressources dialectales françaises. Ainsi recourt-il au patois auvergnat pour le personnage du commissaire Fumi. La traduction de Bonalumi a été diversement reçue. Si Gadda lui-même en pensait le plus grand bien, si un Daniel Pennac par exemple vante Gadda pour la saveur de sa langue, qu'il lit en traduction, Paul Renucci a critiqué la traduction de Gadda, dont il déplore l'absence de systématisme et le recours ponctuel aux dialectes français qui, du fait du recul des dialectes plus précoce en France qu'en Italie, ne sauraient servir d'équivalents. II préconise de jouer sur la différence des statuts sociaux, de travailler plutôt sur les sociolectes, les niveaux de langue. Le problème d'une telle proposition est la suivante, pour Giovanni Clerico qui critique Renucci : c'est réduire les dialectes à des sociolectes et faire disparaître la composante géographique de leur usage. Or l'" affreux pastis ", le "pasticciaccio " - qui au passage n'est pas une anisette mais une tourte, un pâté fait d'éléments composites - ce n'est pas seulement le crime sur lequel enquête Ingravallo, mais c'est aussi le mélange linguistique italien. Et au-delà, traduire ainsi, c'est en un sens, naturaliser le texte, en faire une histoire de truands français et non italiens, commettre, donc, la faute d'ethnocentrisme tant critiquée par Antoine Berman(1).

C'est pourtant la solution que choisit Michèle Causse, la traductrice de l'ouvrage de Natalia Ginzburg, Lessico famigliare - traduit sous le titre "Les mots de la tribu ». Le titre italien l'annonce : il s'agit de construire des souvenirs d'enfance en se fondant sur les idiolectes familiaux, entre bons mots devenus proverbiaux et régionalismes divers. Le passage suivant évoque des anecdotes racontées par la mère de la narratrice, qui rapportait les expressions en dialecte milanais entendues dans la rue :

Mia madre era milanese, ma di origine triestina anche lei; e d'altronde aveva sposato, con mio padre, anche molte espressioni triestine. II milanese veniva a mescolarsi nel suo parlare, quando raccontava ricordi d'infanzia.

Aveva visto un giorno, camminando per strada, a Milano, quand'era piccola, un signore impettito, immobile davanti a una vettrina di parrucchiere, che fissava una testa di bambola, e diceva tra sé:

(1) Voir notamment Antoine Berman, "La traduction et la lettre - ou l'auberge du lointain ", dans Les Tours de Babel (Essais sur la traduction). Antoine Berman y écrit notamment ceci, à propos de la traduction des proverbes, et que l'on peut transposer au problème de la traduction des régionalismes "Or, même si le sens est identique, remplacer un idiotisme par son équivalent est un ethnocentrisme qui, répété à une grande échelle, aboutirait à cette absurdité que, dans Typhon, les personnages s'exprimeraient avec des images françaises ! [...] Les équivalents d'une locution ou d'un proverbe ne les remplacent pas. Traduire n'est pas chercher des équivalences. En outre, vouloir les remplacer est ignorer qu'il existe en nous une conscience-de-proverbe qui percevra tout de suite, dans un nouveau proverbe, le frère d'un proverbe du cru. " (Article cité, p. 80) 
- Bella, bella, bella. Troppo lunga de col.

Molti dei suoi ricordi erano cosi: semplici frasi che aveva sentito. Un giorno, con le sue compagne di collegio e con le maestre, era fuori a passeggio. D'un tratto una delle bambine s'era staccata dalla fila, correndo ad abbracciare un cane che passava; lo abbracciava, e diceva:

- L'è le, l'è le, l'è la sorella della mia cagna!

Ma mère était milanaise, mais d'origine triestine, elle aussi, d'ailleurs elle avait épousé, en même temps que mon père, bon nombre d'expressions triestines. Le milanais venait se mélanger à ses propos quand elle évoquait des souvenirs d'enfance.

Elle avait vu un jour, en se promenant dans la rue, à Milan, alors qu'elle était toute petite, un monsieur guindé, immobile devant une vitrine de coiffeur, qui murmurait en regardant une tête de poupée :

- Belle, belle, belle. Trop longue de cou.

Ses souvenirs étaient pour une bonne part composés de simples phrases qu'elle avait entendues. Un jour, elle était en promenade avec ses camarades de collège. Tout à coup l'une de ses camarades sortit des rangs et courut embrasser un chien qui passait.

- La v'là, la v'là, la soeur de ma chienne! (N. Ginzburg traduite par Causse, 1963, p. 16)

Dans le texte italien de Ginzburg, les paroles rapportées par la mère « Bella, bella, bella. Troppo lunga de col " et "L'è le, l'è le, l'è la sorella della mia cagna ! " laissent apparaître, quoi que discrètement, la prononciation milanaise. Sur de si courtes phrases, la marge de manœuvre de la traductrice était étroite. Aussi a-t-elle marqué le contraste des propos rapportés et de la narration en accentuant leur aspect oral - surtout dans la deuxième phrase, où elle élide une partie du mot "voilà ", ce qui en un sens peut former un équivalent, par transposition, avec la prononciation milanaise. Mais le lecteur français sait qu'il s'agit de milanais uniquement parce que la narratrice l'a signalé plus haut.

II faut dire que rendre le milanais en français - ou tout autre dialecte - peut sembler une contradiction dans les termes. Si on peut imaginer une traduction qui cite des mots du texte original afin d'en rendre la saveur, comment imaginer une traduction qui, traduisant, rende les inflexions dialectales - et notamment leur prononciation à quoi on les reconnaît en littérature, dans la mesure où la graphie italienne est modifiée - tout en traduisant en langue française ?

Le pari de Serge Quadruppani, traducteur de Camilleri, est précisément de laisser entendre le sicilien dans le français, et surtout de laisser entendre dans le français la différence entre sicilien et italien standard, sans pour autant rapporter le sicilien à un registre bas, et l'italien à un registre haut. Camilleri, comme Gadda en un sens, n'écrit pas un sicilien " pur " (si tant est qu'une telle chose existe) mais une langue italienne sicilianisée. Dans les dialogues en particulier, il adopte la syntaxe sicilienne (utilisation du passé simple, ordre Sujet-Objet-Verbe entre autres) et surtout une graphie qui tienne compte de la prononciation de la région d'Agrigente. Un très court exemple issu du Tour de la bouée - c'est le personnage de Catarella, le réceptionniste, qui prend la parole : 
"L'attrovai! L'intintificazioni fecil» urlò Catarella avanzando e posando sulla scrivania una foto ingrandita e una scheda stampata dal computer. (p. 94)

- Je l'attrouvai ! L'itintification je fis ! hurla Catarella en avançant pour poser sur le bureau une photo agrandie et le tirage d'une fiche de l'ordinateur. (p. 90)

Cet exemple, pour être court, n'en est pas moins représentatif de la méthode de Quadruppani, à l'échelle du roman en entier, et de ses autres traductions. On voit dans ces quelques mots comment, d'un point de vue syntaxique, Quadruppani calque les structures siciliennes, précisément dans la mesure où elles représentent déjà un écart par rapport à l'italien standard, et même si elles ne correspondent à aucun régionalisme connu en français. Par ailleurs, lorsque la racine des mots français est suffisamment proche de celle des mots italiens, Quadruppani déforme la graphie - et mentalement la prononciation - française, sur le modèle des déformations phonétiques de l'italien. Dans l'exemple cité on trouve ainsi un " a " épenthétique suivi d'une consonne au début du mot "attrouvai ", pour " trouvai ", sur le modèle de trovai > attrovai.

Par contraste avec les exemples des traductions de Gadda et de Ginzburg cités plus haut, la nouveauté apportée par Quadruppani réside dans la tentative de traduire en français - nul mot italien à l'échelle de ces quelques lignes, même s'il y en a bon nombre à l'échelle de la traduction française - tout en rendant sensible l'hétérogénéité linguistique du texte source, sans pour autant transposer l'univers sicilien dans une région française - pas d'accent marseillais, par exemple. Ce choix suppose de rompre avec une convention tacite de la traduction, respectée par les deux traducteurs de Gadda et de Ginzburg : l'impératif de traduire dans un français entièrement français - pas nécessairement soutenu, mais du moins, préexistant. Or Quadruppani invente un français inouï, un français sicilianisé, réduisant ainsi ce qui pouvait sembler intraduisible : l'ancrage régional des dialectes italiens.

II ne faut pas voir là simplement un choix de traducteur engageant une vision du texte étranger, et il serait absurde de se contenter de dire que Quadruppani a raison là où Causse et Bonalumi ont tort, parce que le premier rend la traduction à la sicilianité de l'original, tandis que les seconds pratiquent une traduction ethnocentriste, qui tire le texte étranger vers l'univers français. Le travail de Quadruppani s'ancre en effet dans un contexte historique spécifique : il traduit après la parution des ouvrages d'Henri Meschonnic(2) et d'Antoine Berman (notamment Pour une critique des traductions : John Donne), qui affirment d'une part la dimension éthique du travail du traducteur, qui définit un rapport à l'autre, à l'étranger, et d'autre part la possibilité de s'affranchir des limites du "bon français ". De même que les auteurs ont tous les droits pour l'invention linguistique, les traducteurs peuvent faire osciller les limites de la langue française.

(2) Voir notamment les travaux d'Henri Meschonnic, Poétique du traduire ou encore la préface aux Cinq rouleaux, intitulée en guise de manifeste "Pour une poétique de la traduction ", et dans laquelle Meschonnic affirme p. 9 : "Chaque époque d'écriture enrichit la possibilité de traduire. Chaque époque de traduction enrichit la possibilité d'écrire. Le français n'est plus ce qu'il était avant le surréalisme. " 


\section{Traduire ou ne pas traduire?}

Un travail comme celui de Quadruppani est le signe d'une extension des possibilités de la traduction, et de ce fait, d'une forme de recul (ou de redéfinition) des intraduisibles, qui ne semblent l'être non tant du fait de la nature intrinsèque du texte source que des présupposés sur la traduction comme devant être intégrale et rédigée " comme si l'auteur avait écrit directement en français ". Pour autant toute difficulté n'est pas abolie. Venons-en maintenant à un autre type de cas : celui des mots en langue étrangère apparaissant dans la traduction. Plusieurs exemples ici, qui seront tous successivement cités avant que ne soit élaborée une brève analyse.

D'abord, trois extraits du Jardin des Finzi-Contini, le roman de Bassani traduit en 1964 par Michel Arnaud. Dans les deux premiers, Bassani fait entendre les langues juives parlées dans la famille de Micol, l'héroïne

... i fratelli della signora Olga, l'ingegnere delle ferrovie e il tisiologo, parlottare fra loro mezzo in veneto e mezzo in ispagnolo ("Cossa xé stas meldando? Su, Giulio, alevantate, ajde! E procura de far star in pie anca il chico...n), e poi smettere, d'un tratto, e unirsi a voce altissima, in ebraico, alle litanie del rabbino. (Bassani, 1962 : p. 41)

... les frères de la Signora Olga, l'ingénieur des chemins de fer et le phtisiologue, chuchoter entre eux à moitié en vénitien et moitié en espagnol ("Cossa xé che stas meldando? Su, Giulio, alevantate, ajde! E procura de far star in pie anca il chico...) et puis, cessant brusquement de chuchoter, s'unir à voix très haute, en hébreu, aux litanies du rabbin.

(NdT : "Qu'est-ce que tu lis ? Allons, Giulio, lève-toi ! Et tâche de faire rester debout le petit lui aussi...")

(Bassani traduit par Arnaud, 1964 : p. 51)

Ici, Micol explique au narrateur un mot employé par son père :

Le disse che stesse "callada", cioè zitta. Non lo vedeva che c'era il musafir?

Mi chinai all'orecchio di Micol.

"Invece che "sta' callada", noialtri diciamo "sta'sciadok". Ma musafir, che cosa significa?"

"Ospite», mi sussurrò lei di rimando. "Goi, però». (p. 99)

Il lui dit de se tenir callada, c'est-à-dire de se tenir coite. Est-ce qu'elle ne voyait pas qu'il y avait un musafir?

Je me penchai à l'oreille de Micol.

- Au lieu de "Tiens toi callada ", nous autres nous disons "Tiens toi sciadok ". Mais que signifie musafir?

- Hôte, me chuchota-t-elle en retour. Mais hôte goy. (p. 127)

Plus loin dans le roman, la permanence de mots cités en langue étrangère est d'un tout autre ordre. II s'agit des poèmes évoqués par les personnages, qui ne sont jamais traduits en fran- 
çais dans le corps du texte, que ce soit à la fin du roman lorsque le personnage de Malnate cite des poèmes en milanais, ou, ici, lorsque dans un échange épistolaire, Micol propose au narrateur sa traduction italienne d'un poème d'Emily Dickinson :

La lettera tirava avanti per altre due pagine e mezzo, discorrendo della tesi ormai "veleggiante verso il traguardo finale", accennando a Venezia che, d'inverno, faceva "semplicemente piangeren, e chiudendo a sorpresa con la traduzione in versi di una poesia di Emily Dickinson.

Questa:

Morii per la Bellezza: e da poco ero

Discesa nell'avello,

Che, caduto per Vero, uno fu messo

Nell'attiguo sacello.

"Perché sei morta?", mi chiese sommesso.

Dissi: "Morii pel Bello."

"lo per la Verità; dunque è la stesso,

- disse -, son tuo fratello."

Da tomba a tomba, come due congiunti

Incontratisi a notte,

Parlavamo cosi: finché raggiunti

L'erba ebbe nomi e bocche.

Seguiva un poscritto, che diceva testualmente: "Alas, poor Emily. Ecco il genere di compensi su cui è costretto a puntare l'abbietto zitellaggio!n (p. 156-157)

La lettre continuait pendant deux pages et demie encore, parlant de son diplôme "qui voguait maintenant vers le poteau d'arrivée ", disant quelques mots de Venise qui, en hiver, était " tout simplement à pleurer ", et se terminant inopinément par la traduction en vers d'un poème d'Emily Dickinson.

La voici :

Morii per la Bellezza: e da poco ero

Discesa nell'avello,

Che, caduto per Vero, uno fu messo

Nell'attiguo sacello.

"Perché sei morta?», mi chiese sommesso.

Dissi: "Morii pel Bello.»

"lo per la Verità; dunque è la stesso,

- disse -, son tuo fratello."

Da tomba a tomba, come due congiunti

Incontratisi a notte,

Parlavamo cosi: finché raggiunti

L'erba ebbe nomi e bocche.

Venait ensuite un post-scriptum qui disait textuellement : " Alas, poor Emily. Voilà le genre de compensation sur lequel l'abject célibat féminin est contraint de miser !" 
Une note du traducteur, dont l'appel est placé en bas du poème en italien, en donne une traduction, Voici l'intégralité de la note :

Note du traducteur

Cette traduction italienne du poème d'Emily Dickinson étant plutôt une adaptation, il a semblé plus juste d'en donner le texte italien, mais pour que le lecteur puisse juger de la part créatrice de Micol, voici d'abord la traduction littérale de l'original américain :

"Je suis morte pour la beauté, mais à peine étais-je / Installée dans la tombe, / Qu'un qui était mort pour la vérité fut couché / Dans une niche adjacente.

II demanda doucement pourquoi j'avais péri ? / "Pour la beauté ", répondis-je. / "Et moi pour la vérité - les deux ne font qu'un ; / Nous sommes frères ", dit-il.

Et de la sorte, tels des parents se rencontrant la nuit, / Nous parlâmes d'une niche à l'autre, / Jusqu'à ce que la mousse ait atteint nos lèvres, / Et recouvert nos noms. "

Et voici maintenant la traduction littérale de la version italienne de ce poème :

"Je suis morte pour la Beauté ; et j'étais depuis peu / descendue au sépulcre, / quand, tombé pour le Vrai, quelqu'un fut mis / dans le Sacellum contigu ".

"Pourquoi es-tu morte? me demanda-t-il à mi-voix. / Je répondis : " Je suis morte pour le Beau. " / "Moi pour la Vérité : donc, c'est la même chose, / dit-il - je suis ton frère. "

De tombe à tombe, comme deux parents / se rencontrant la nuit, / nous parlions ainsi ; jusqu'à ce qu'atteint / eut l'herbe nos noms et nos bouches. ". (p. 197-198)

Un court passage du Tour de la bouée d'Andrea Camilleri, traduit par Serge Quadruppani, représentatif de la façon dont les proverbes et dictons siciliens sont traités par l'auteur et son traducteur :

"All'annigatu petri di "noddru" era il detto popolare che veniva esclamato quando una insopportabile serie di disgrazie s'abbatteva su qualche spinturato. (p. 9)

All'anigatu, petri di 'ncoddru : " au noyé, une pierre par derrière ", tel était le dicton populaire qu'on lançait quand une insupportable série de déveines s'abattait sur quelque malheureux. (p. 11)

En contrepoint maintenant un extrait de Montedidio du napolitain Erri De Luca, traduit en français par Danièle Valin, texte et traduction étant à peu de choses près contemporains de l'exemple de Camilleri :

Sento strilli e voci napoletane, parlo napoletano, però scrivo italiano. "Stiamo in Italia, dice babbo, ma non siamo italiani. Per parlare la lingua la dobbiamo studiare, è come all'estero, come in America, ma senza andarsene. Molti di noi non lo parleranno mai l'italiano e moriranno in napoletano. È una lingua difficile, dice, ma tu l'imparerai e sarai italiano. lo e mamma tua no, noi nun pu, nun po, nuie nun putimmo." Vuole dire "non possiamo" ma non gli esce il verbo. Glielo dico, "non possiamo", bravo, dice, bravo, tu conosci la lingua nazionale. Si la conosco e di nascosto la scrivo pure e mi sento un poco traditore del napoletano e allora in testa mi recito il suo verbo potere: i' pozzo, tu puozze, isso po', nuie putimmo, vuie putite, lloro ponno. Mamma non è d'accordo con babbo, lei dice: "Nuie simmo napulitane e basta". Ll'Italia mia, dice con due 
elle di articolo, Il'talia mia sta in America, addo ce vive meza famiglia mia. "'A patria è chella ca te dà a magna'." dice e conclude. Babbo per scherzare le risponde: "Allora 'a patria mia si' tu". (De Luca, 2001 : p. 20)

J'entends des cris et des voix napolitaines, je parle napolitain, mais j'écris en italien. "Nous vivons en Italie, dit papa, mais nous ne sommes pas italiens. Pour parler la langue, nous devons l'étudier, c'est comme à l'étranger, comme en Amérique, mais sans s'en aller. Beaucoup d'entre nous ne le parleront jamais l'italien et ils mourront en napolitain. C'est une langue difficile, mais tu l'apprendras et tu seras italien. Ta maman et moi non, noi nun pu, nun puo, nuie nun putimmo. " II veut dire "nous ne pouvons pas", mais le verbe ne lui vient pas. Je le lui dis " nous ne pouvons pas ", bravo, dit-il, bravo, toi tu connais la langue nationale. Oui, je la connais, je l'écris même en cachette, et je me sens un peu traître au napolitain, alors je me récite mentalement son verbe pouvoir : i' pozzo, tu puozze, isso po', nuie putimmo, vuie putite, lloro ponno. Maman n'est pas d'accord avec papa, elle dit : " nous sommes napolitains, un point c'est tout. " Mmon Italie, dit-elle avec deux m, mmon Italie est en Amérique, là où vit la moitié de ma famille. La patrie c'est celle qui te donne à manger, dit-elle en concluant. Papa lui répond pour blaguer : "Alors ma patrie à moi c'est toi ". (De Luca traduit par Valin, 2002 : p. 28-29)

Je proposais au début de l'article d'appeler les mots importés du texte original des « intraduits " plutôt que les intraduisibles. Tout est relatif - les mots étrangers figurant dans ces extraits de traduction sont le plus souvent traduits d'une façon ou d'une autre, même si la persistance de mots étrangers dans la traduction française met à mal le postulat de départ qui supposait que le propre de la traduction littéraire est de tout traduire en ne laissant rien subsister de la langue de départ. Or nous constatons que dans ces quelques extraits - qui sont globalement représentatifs de la façon dont ces ouvrages sont traduits - que les traducteurs ont introduit dans leur texte français une forme de redoublement, de feuilletage de la traduction.

Un premier cas est représenté par la non-traduction dans le corps de la page de la version italienne, faite dans le roman par le personnage de Micol, du poème de Dickinson. Le traducteur Michel Arnaud en effet, laisse la traduction italienne du poème anglais. En note en revanche il ne propose pas une, mais deux traductions du poème : la première " littérale ", faite directement sur l'américain, la seconde une traduction française de la traduction italienne, permettant de juger du travail de Micol qui, selon lui, confine davantage à l'adaptation qu'à la traduction. Pourquoi ne pas traduire directement dans le corps du texte? Plusieurs possibilités d'interprétation ici : d'abord on touche peut-être à l'idée selon laquelle la poésie est, sinon intraduisible, du moins impossible à traduire entièrement, en recréant à l'identique sens et forme. Peut-être, puisqu'il s'agit justement d'évaluer la vision qu'a Micol de la traduction, le but est-il de ne pas recouvrir le travail de Micol - en réalité, de Bassani - par une nouvelle traduction, fût-elle effectuée dans le même esprit. Toujours est-il que l'effet pour le lecteur français est bien différent de celui ressenti par le lecteur italien qui n'a affaire qu'à une traduction, celle de Micol, là où le lecteur français se trouve confronté à trois textes différents - paradoxalement, le texte américain de Dickinson n'est pas cité. Le paradoxe de la traduction d'Arnaud est qu'elle est rédigée à une époque contemporaine de celle de Gadda par 
Bonalumi, où il est d'usage de tout traduire, d'invisibiliser au maximum le travail du traducteur. Le cas de Michel Arnaud montre avec ce contre-exemple à quel point on ne saurait généraliser de façon absolument déterministe sur les âges de la traduction.

Un deuxième type de non-traduction concerne le maintien de mots en langue étrangère dans la traduction française, pour rendre compte de l'insertion dans le texte littéraire de mots qui sont eux-mêmes distincts de l'italien standard : mélange de vénitien et de judéo-espagnol, et mots d'origine hébraïque constituant les idiolectes familiaux des Finzi-Contini chez Bassani ; mots et phrases napolitains chez Erri De Luca. Ce que l'on constate ici, que l'on observe du reste aussi dans les ouvrages de Camilleri traduits par Quadruppani, c'est que là où le texte italien ne traduit pas systématiquement les passages dialectaux, la traduction, elle, conserve le dialecte tel quel - le cas échéant avec une mise en forme spécifique - puis le fait suivre d'une traduction, immédiatement dans la traduction de Camilleri par Quadruppani, en notes de bas de page dans la traduction de Bassani par Michel Arnaud. Ces auteurs, à d'autres moments de l'œuvre, glosent dans le texte italien même le passage en dialecte de manière à le rendre compréhensible du lecteur italien, mais dans les deux extraits concernés, le dialecte est laissé sans glose italienne : on peut supposer que les auteurs s'attendent à ce qu'un lecteur italophone comprenne assez bien le sens de la phrase, tout en percevant la particularité de la langue. On ne peut cependant attendre une telle compétence d'un lecteur francophone, d'où l'élaboration d'un système qui permette à la fois de conserver l'hétérogénéité linguistique du texte littéraire, et la compréhension par le lecteur.

Pour autant, Danièle Valin, la traductrice d'Erri De Luca, ne procède pas exactement de la même façon. Comme Quadruppani, elle s'abstient d'utiliser des notes de bas de page. En revanche, elle n'ajoute pas de glose en français des extraits en napolitain lorsque le texte italien ne glose pas lui-même. Ainsi, dans le passage cité plus haut, le dialecte napolitain est soit laissé tel quel sans traduction - lorsque le narrateur se récite le verbe " pouvoir " en napolitain, soit traduit dans un français napolitanisé, si dire se peut. Danièle Valin recourt alors au procédé que nous avions observé chez Camilleri traduit par Quadruppani : elle déforme la graphie du français par redoublement de la première consonne du mot, sur le modèle de la prononciation italienne. Mais le procédé est bien moins répandu à l'échelle de sa traduction que chez Quadruppani.

Revenons sur le terme de "feuilletage " de la traduction que j'avais employé plus haut. Je l'emprunte à Michel de Certeau, qui définit ainsi, dans son article "L'idée de traduction de la Bible au XvIIe siècle ", la coexistence dans le texte traduit de plusieurs interventions hétérogènes du traducteur (traduction, glose, note de bas de page dans le corpus italophone qui nous concerne). Certeau appelle de ce terme la façon dont les traducteurs de la Bible de Port-Royal traduisent le texte biblique en manifestant la nature seconde de la traduction. Les procédés utilisés " consistent à réintroduire de l'obscurité sur les bords de la clarté, à créer un tremblé de la traduction même. Ce sera en adjoignant à la version retenue les sens différents dont l'original est susceptible, ou en faisant apparaître un feuilletage de l'original par la traduction 
des variantes hébraïques, grecques ou latines du même passage. Dans la mise en page, une combinaison spatiale du texte central et des marges (ou notes) compense l'excessive clarté du discours continu par une proliférante pluralisation sémantique ou critique. "

Ces mots de Certeau me permettent de conclure en tentant de comparer les intraduits - ou du moins, les mots cités en langue étrangère - dans les ouvrages littéraires et dans les textes des sciences humaines. La traduction du roman de Bassani par Michel Arnaud, avec ses italiques, ses incises, ses notes de bas de pages, procède dans une certaine mesure du feuilletage du texte. Dans une moindre mesure, les traductions des romans de Camilleri et d'Erri De Luca proposent ce feuilletage par la présence de mots de l'original, souvent glosés, qui en tout cas manifestent la nature seconde de la traduction. Ces intraduits ne sont donc ni intraduisibles, ni complètement intraduits, et en ce sens ils répondent, par les diverses solutions élaborées par les traducteurs pour en rendre compte tout en laissant entendre l'original, à la définition que donne Barbara Cassin des intraduisibles dans la présentation du Vocabulaire européen des philosophies :

Parler d'intraduisibles n'implique nullement que les termes en question, ou les expressions, les tours syntaxiques et grammaticaux, ne soient pas traduits et ne puissent pas l'être - l'intraduisible, c'est plutôt ce qu'on ne cesse pas de (ne pas) traduire. Mais cela signale que leur traduction, dans une langue ou dans une autre, fait problème, au point de susciter parfois un néologisme ou l'imposition d'un nouveau sens sur un vieux mot : c'est un indice de la manière dont, d'une langue à l'autre, tant les mots que les réseaux conceptuels ne sont pas superposables. (Cassin, 2004)

La raison de ne pas traduire, ou de ne pas cesser de ne pas traduire, n'est certes pas complètement identique dans les cas des ouvrages que j'ai cités et dans les sciences humaines. Stylistiquement, elle ne prend pas toujours les mêmes formes. Ainsi observe-t-on, dans le chapitre "La conversion des juifs de Minorque " dans Le Fil et les traces de Carlo Ginzburg, la présence en italien comme dans la traduction française de mots anglais de l'ouvrage de Peter Brown que Ginzburg commente et analyse(3). Le travail du traducteur n'est pas formellement

(3) On lit dans le texte de Ginzburg : " "Il lettore deve perdonarmi”, scrive Brown, "se, nel descrivere una faccenda molto sudicia [...] mi limiterò ad assumere la prospettiva del vescovo Severo, nostra unica fonte, e a parlare del patrocinium di santo Stefano come di un potere 'incontaminato' [clean]". II problema di metodo sollevato da Brown è ovviamente molto serio. Ma le parole lievamente ambigue che ho appena citato potrebbero indurre qualche lettore a intendere, erroneamente, che categorie come clean e unclean, "incontaminato" e "contaminato", derivano dalla fonte stessa. " (II filo e le tracce. Vero falso finto, Milano, Feltrinelli, 2006, p. 40), et dans la traduction française de Martin Rueff, "Le lecteur doit me pardonner, écrit Brown, si, pour décrire une histoire aussi dégoûtante, je me limite à la perspective de l'évêque Sévère, notre unique source, et si je parle du patrocinium de saint Étienne comme un pouvoir pur [clean]. " Le problème de méthode que pose Brown est évidemment des plus sérieux. Mais les propos légèrement ambigus que je viens de citer pourraient amener quelques lecteurs à supposer, à tort, que des catégories comme "clean " et " unclean ", "pur " et " impur ", viennent de la source elle-même ". 
identique à celui d'un Quadruppani : le mot anglais est placé après l'italien, entre parenthèses, à titre de précision. Mais pour autant il n'y a pas de nette distinction entre les raisons de citer en langue étrangère en littérature et en sciences humaines, et l'effet reste celui du feuilletage, qui laisse apparaître la nature seconde, relative de la traduction.

Les cas évoqués ici ne sont pas pour autant généralisables : je me suis fondée sur des exemples de textes littéraires présentant des phénomènes de diglossie ou de pluriglossie, mais il est évident que ce problème-là disparaît si l'ouvrage à traduire est rédigé dans une langue homogène. Mais précisément cet exemple semble indiquer qu'il n'est pas possible d'affirmer que la différence entre traduction des textes de SHS et de littérature soit claire. Dans un cas comme dans l'autre, il s'agit de rendre un discours - et de même qu'on ne traduit pas de la même façon Camilleri et Manzoni, on ne traduit pas de la même façon Heidegger et Benjamin.

\section{Bibliographie}

\section{- Ouvrages littéraires cités}

BASSANI Giorgio, 1962, II giardino dei Finzi-Contini, Milano (Italie), Einaudi.

BASSANI Giorgio, 1964, Le Jardin des Finzi-Contini, traduit de l'italien par Michel Arnaud, Paris, Gallimard.

CAMILLERI Andrea, 2005, Le Tour de la bouée, traduit de l'italien (Sicile) par Serge Quadruppani, Paris, Pocket.

DE LUCA Erri, 2001, Montedidio, Milano (Italie), Feltrinelli.

DE LUCA Erri, 2002, Montedidio, traduit de l'italien par Danièle Valin, Paris, Gallimard.

GADDA, Carlo Emilio, 1957, Quer pasticciaccio brutto de via Merulana, Milano (Italie), Garzanti.

GADDA Carlo Emilio, 1963, L'affreux pastis de la rue des Merles, traduit de l'italien par Louis Bonalumi, Paris, Seuil.

GINZBURG Natalia, 1963, Lessico famigliare, Torino (Italie), Einaudi.

GINZBURG Natalia, 1963, Les Mots de la tribu, traduit de l'italien par Michèle Causse, Paris, Grasset.

GINZBURG Carlo, 2006, Il filo e le tracce. Vero falso finto, Milano (Italie), Feltrinelli, 2006. 


\section{- Littérature critique}

BERMAN Antoine Berman, 1995, Pour une critique des traductions : John Donne, Paris, Gallimard. BERMAN Antoine, 1985, "La traduction et la lettre - ou l'auberge du lointain ", dans Les Tours de Babel (Essais sur la traduction), Éditions Trans-Europ-Repress.

CASSIN Barbara (dir.), 2004, Vocabulaire européen des philosophies. Dictionnaire des intraduisibles, Éditions du Seuil / Dictionnaires Robert.

CERTEAU Michel de, 1978, "L'idée de traduction de la Bible au XVIIe siècle ", dans Recherche de science religieuse (66).

CLERICO Giovanni, 2007 "Le détail et l'ensemble : Gadda et la traduction ", in The Edinburgh Journal of Gadda Studies,

http://www.gadda.ed.ac.uk/Pages/journal/supp5archivm/ragioni/ragioniclerico.php consulté le 27 février 2015.

MESCHONNIC Henri, 1970, Les Cinq Rouleaux, Paris, Gallimard.

MESCHONNIC Henri, 1999, Poétique du traduire, Lagrasse, Verdier.

Claire Placial est maître de conférences en littérature comparée à l'université de Lorraine. Ses recherches portent principalement sur l'histoire des théories et des pratiques de la traduction, notamment des textes sacrés. Elle s'intéresse par ailleurs à l'histoire des théories linguistiques, particulièrement aux notions d'esprit des langues et de génie des langues. Outre ses activités universitaires, elle traduit des textes littéraires depuis l'allemand (Goethe, Heine) et l'italien. 